Turk. J. Math. Comput. Sci.

$12(2)(2020) 68-75$

(C) MatDer

DOI : $10.47000 /$ tjmcs. 701638

\title{
Bi-Periodic Balancing Quaternions
}

\author{
Emre Sevgi ${ }^{1, *(D)}$, Dursun Taşci ${ }^{1}$ (iD \\ ${ }^{1}$ Department of Mathematics, Faculty of Science, Gazi University, 06500, Ankara, Turkey.
}

Received: 10-03-2020 • Accepted: 17-07-2020

АвstRact. In this paper, we first define the bi-periodic balancing quaternions. We give the generating function and Binet formula for this quaternion. Then, we obtain some identities and properties including this quaternion.

2010 AMS Classification: 11B37, 11B39, 20G20

Keywords: Bi-periodic balancing numbers, Binet formula, Generating function, Cassini identity, Catalan identity.

\section{INTRODUCTION}

Quaternion is a number system which extends the complex numbers and defined by Hamilton as

$$
q=a_{0} e_{0}+a_{1} e_{1}+a_{2} e_{2}+a_{3} e_{3}
$$

where $e_{1}, e_{2}, e_{3}$ are standart basis in $\mathbb{R}^{3}, e_{0}=1$ and $a_{0}, a_{1}, a_{2}, a_{3}$ are real numbers [4].

There are so many researches on integer sequences such as Fibonacci, Lucas and Jacobsthal sequences [7-9, 11]. By the view of the definition of quaternions, the quaternions of some integer sequences was defined. Horadam [5] gave the definition of Fibonacci and Iyer [6] gave the definition of Lucas quaternions, respectively

$$
Q_{n}=e_{0} F_{n}+e_{1} F_{n+1}+e_{2} F_{n+2}+e_{3} F_{n+3}
$$

and

$$
T_{n}=e_{0} L_{n}+e_{1} L_{n+1}+e_{2} L_{n+2}+e_{3} L_{n+3}
$$

where $F_{n}$ is the $n$-th Fibonacci number and $L_{n}$ is the $n$-th Lucas number.

Also, Halic1 [3] gave the generating functions and Binet formulas for Fibonacci and Lucas quaternions. Finally, Tan et al. [10] introduced the bi-periodic Fibonacci quaternions which is a new generalization of the Fibonacci quaternions as

$$
Q_{n}=\sum_{l=0}^{3} q_{n+l} e_{l}, \quad n \geq 0
$$

where $q_{n}$ is the $n$-th bi-periodic Fibonacci number which was defined by Edson and Yayenie [2].

In the second section, we will give the definition of bi-periodic balancing quaternion. Also, we will obtain the Binet formula, the generating function and some identities of this quaternion. Before these, we will recall the balancing numbers and bi-periodic balancing numbers.

*Corresponding Author

Email addresses: emresevgi@gazi.edu.tr (E. Sevgi),dtasci@gazi.edu.tr (D. Tasci) 
Balancing numbers $\left\{d_{n}\right\}_{n=0}^{\infty}$ was defined by Behera and Panda [1] with initial conditions $d_{0}=0, d_{1}=1$ and the recurrence relation

$$
d_{n}=6 d_{n-1}-d_{n-2}, \quad n \geq 2 .
$$

Definition 1.1. For any two non-zero real numbers $c$ and $d$, the bi-periodic balancing numbers $\left\{b_{n}\right\}_{n=0}^{\infty}$ is defined for $n \geq 2$ with initial conditions $b_{0}=0, b_{1}=1$ and the recurrence relation

$$
b_{n}= \begin{cases}6 c b_{n-1}-b_{n-2}, & \text { if } n \text { is even } \\ 6 d b_{n-1}-b_{n-2}, & \text { if } n \text { is odd } .\end{cases}
$$

When $c=d=1$, we have the classic balancing numbers. If we set $c=d=k$, for any positive number, we get the $k$-balancing numbers. The first five elements of the bi-periodic balancing numbers are

$$
b_{0}=0, b_{1}=1, b_{2}=6 c, b_{3}=36 c d-1, b_{4}=216 c^{2} d-12 c .
$$

The quadratic equation for the bi-periodic balancing numbers is defined as

$$
x^{2}-6 c d x+c d=0
$$

with roots

$$
\alpha=3 c d+\sqrt{9 c^{2} d^{2}-c d} \text { and } \beta=3 c d-\sqrt{9 c^{2} d^{2}-c d} .
$$

Lemma 1.2. We can express the terms of the bi-periodic balancing numbers $\left\{b_{n}\right\}_{n=0}^{\infty}$ by using the Binet formula;

$$
b_{m}=\left(\frac{c^{1-\xi(m)}}{(c d)^{\left\lfloor\frac{m}{2}\right\rfloor}}\right) \frac{\alpha^{m}-\beta^{m}}{\alpha-\beta}
$$

where $\lfloor c\rfloor$ is the floor function of $c$ and $\xi(m)=m-2\left\lfloor\frac{m}{2}\right\rfloor$ is the parity function.

Lemma 1.3. The bi-periodic balancing numbers $\left\{b_{n}\right\}_{n=0}^{\infty}$ satisfies the following properties:

$$
\begin{gathered}
b_{2 n}=(36 c d-2) b_{2 n-2}-b_{2 n-4}, \\
b_{2 n+1}=(36 c d-2) b_{2 n-1}-b_{2 n-3} .
\end{gathered}
$$

Proof. The proof can be seen by the recurrence relation of bi-periodic balancing numbers.

\section{Main Results}

Definition 2.1. For $n \geq 0$, the bi-periodic balancing quaternions $B_{n}$ are defined as

$$
B_{n}=\sum_{l=0}^{3} b_{n+l} e_{l}
$$

where $b_{n}$ is the $n$-th bi-periodic balancing number.

Theorem 2.2. The generating function for the bi-periodic balancing quaternion $B_{n}$ is

$$
G(t)=\frac{B_{0}+\left(B_{1}-6 d B_{0}\right) t+6(c-d) S(t)}{1-6 d t+t^{2}}
$$

where

$$
\begin{gathered}
S(t)=\operatorname{tg}(t) e_{0}+(g(t)-t) e_{1}+\left(\frac{g(t)}{t}-1\right) e_{2}+\left(\frac{g(t)-t-(36 c d-1) t^{3}}{t^{2}}\right) e_{3}, \\
g(t)=\sum_{n=1}^{\infty} b_{2 n-1} t^{2 n-1}=\frac{t+t^{3}}{1-(36 c d-2) t^{2}+t^{4}} .
\end{gathered}
$$


Proof. The formal power series representation of the generating function for $B_{n}$ is

$$
G(t)=B_{0}+B_{1} t+B_{2} t^{2}+\cdots+B_{r} t^{r}+\cdots=\sum_{n=0}^{\infty} B_{n} t^{n} .
$$

By multiplying this series by $6 d t$ and $t^{2}$ respectively, we can get the following series;

$$
6 d t G(t)=6 d B_{0} t+6 d B_{1} t^{2}+6 d B_{2} t^{3}+\cdots+6 d B_{r} t^{r+1}+\cdots
$$

and

$$
t^{2} G(t)=B_{0} t^{2}+B_{1} t^{3}+B_{2} t^{4}+\cdots+B_{r} t^{r+2}+\cdots .
$$

Since $b_{2 k+1}=6 d b_{2 k}-b_{2 k-1}$ and $b_{2 k}=6 c b_{2 k-1}-b_{2 k-2}$, we can write

$$
\begin{aligned}
\left(1-6 d t+t^{2}\right) G(t)= & B_{0}+\left(B_{1}-6 d B_{0}\right) t+\sum_{n=2}^{\infty}\left(B_{n}-6 d B_{n-1}+B_{n-2}\right) t^{n} \\
= & B_{0}+\left(B_{1}-6 d B_{0}\right) t+6(c-d) t\left(\sum_{n=1}^{\infty} b_{2 n-1} t^{2 n-1}\right) e_{0} \\
& +6(c-d)\left(\sum_{n=2}^{\infty} b_{2 n-1} t^{2 n-1}\right) e_{1}+6(c-d)\left(\sum_{n=1}^{\infty} b_{2 n+1} t^{2 n}\right) e_{2} \\
& +6(c-d)\left(\sum_{n=2}^{\infty} b_{2 n+1} t^{2 n-1}\right) e_{3} .
\end{aligned}
$$

Now we define $g(t)$ as

$$
g(t)=\sum_{n=1}^{\infty} b_{2 n-1} x^{2 n-1}
$$

By writing (2.2) in the above expansion, we get

$$
\begin{aligned}
\left(1-6 d t+t^{2}\right) G(t)= & B_{0}+\left(B_{1}-6 d B_{0}\right) t+6(c-d) t g(t) e_{0} \\
& +6(c-d)(g(t)-t) e_{1}+6(c-d)\left(\frac{g(t)}{t}-1\right) e_{2} \\
& +6(c-d)\left(\frac{g(t)-t-(36 c d-1) t^{3}}{t^{2}}\right) e_{3}
\end{aligned}
$$

Now, we define $S(t)$ series as

$$
S(t)=\operatorname{tg}(t) e_{0}+(g(t)-t) e_{1}+\left(\frac{g(t)}{t}-1\right) e_{2}+\left(\frac{g(t)-t-(36 c d-1) t^{3}}{t^{2}}\right) e_{3} .
$$

By writing $S(t)$ in equation (2.3), we get

$$
\left(1-6 d t+t^{2}\right) G(t)=B_{0}+\left(B_{1}-6 d B_{0}\right) t+6(c-d) S(t) .
$$

Simplifying the above equation, we get the generating function as

$$
G(t)=\frac{B_{0}+\left(B_{1}-6 d B_{0}\right) t+6(c-d) S(t)}{1-6 d t+t^{2}}
$$

By Lemma 1.3, we have $b_{2 n-1}=(36 c d-2) b_{2 n-3}-b_{2 n-5}$, so we get

$$
\left(1-(36 c d-2) t^{2}+t^{4}\right) g(t)=\sum_{n=1}^{\infty} b_{2 n-1} t^{2 n-1}-(36 c d-2) \sum_{n=2}^{\infty} b_{2 n-3} t^{2 n-1}+\sum_{n=3}^{\infty} b_{2 n-5} t^{2 n-1} .
$$


By using the above expansion, we have

$$
\begin{aligned}
\left(1-(36 c d-2) t^{2}+t^{4}\right) g(t) & =b_{1} t+b_{3} t^{3}-(36 c d-2) b_{1} t^{3}+\sum_{n=3}^{\infty}\left(b_{2 n-1}-(36 c d-2) b_{2 n-3}+b_{2 n-5}\right) t^{2 n-1} \\
& =t+t^{3}
\end{aligned}
$$

Therefore, we hold

$$
g(t)=\sum_{n=1}^{\infty} b_{2 n-1} x^{2 n-1}=\frac{t+t^{3}}{1-(36 c d-2) t^{2}+t^{4}} .
$$

Theorem 2.3. We can express the terms of the bi-periodic balancing quaternions $\left\{B_{n}\right\}_{n=0}^{\infty}$ by using the Binet formula;

$$
B_{n}= \begin{cases}\frac{1}{(c d)^{\left\lfloor\frac{n}{2}\right\rfloor}}\left(\frac{\alpha^{*} \alpha^{n}-\beta^{*} \beta^{n}}{\alpha-\beta}\right), & \text { if } n \text { is even } \\ \frac{1}{(c d)^{\left\lfloor\frac{n}{2}\right\rfloor}}\left(\frac{\alpha^{* *} \alpha^{n}-\beta^{* *} \beta^{n}}{\alpha-\beta}\right), & \text { if } n \text { is odd }\end{cases}
$$

where $\alpha$ and $\beta$ are the roots of quadratic equation of bi-periodic balancing numbers in (1.1), $\lfloor c\rfloor$ is the floor function of $c$ and $\xi(m)=m-2\left\lfloor\frac{m}{2}\right\rfloor$ is the parity function and

$$
\begin{aligned}
& \alpha^{*}=\sum_{l=0}^{3} \frac{c^{\xi(l+1)}}{(c d)^{\left\lfloor\frac{l}{2}\right\rfloor}} \alpha^{l} e_{l}, \quad \beta^{*}=\sum_{l=0}^{3} \frac{c^{\xi(l+1)}}{(c d)^{\left\lfloor\frac{l}{2}\right\rfloor}} \beta^{l} e_{l}, \\
& \alpha^{* *}=\sum_{l=0}^{3} \frac{c^{\xi(l)}}{(c d)^{\left\lfloor\frac{l+1}{2}\right\rfloor}} \alpha^{l} e_{l}, \quad \beta^{* *}=\sum_{l=0}^{3} \frac{c^{\xi(l)}}{(c d)^{\left\lfloor\frac{1+1}{2}\right\rfloor}} \beta^{l} e_{l} .
\end{aligned}
$$

Proof. By using the definition of bi-periodic balancing quaternions (2.1) and Binet formula of bi-periodic balancing numbers (1.2) for $n$ is even, we can write

$$
\begin{aligned}
B_{n}= & \sum_{l=0}^{3} b_{n+l} e_{l} \\
= & \sum_{l=0}^{3}\left(\frac{c^{1-\xi(n+l)}}{(c d)^{\left.\frac{n+l}{2}\right\rfloor}}\right)\left(\frac{\alpha^{n+l}-\beta^{n+l}}{\alpha-\beta}\right) e_{l} \\
= & \frac{c}{(c d)^{\left\lfloor\frac{n}{2}\right\rfloor}}\left(\frac{\alpha^{n}-\beta^{n}}{\alpha-\beta}\right) e_{0}+\frac{1}{(c d)^{\left\lfloor\frac{n+1}{2}\right\rfloor}}\left(\frac{\alpha^{n+1}-\beta^{n+1}}{\alpha-\beta}\right) e_{1} \\
& +\frac{c}{(c d)^{\left.\frac{n+2}{2}\right\rfloor}}\left(\frac{\alpha^{n+2}-\beta^{n+2}}{\alpha-\beta}\right) e_{2}+\frac{1}{(c d)^{\left\lfloor\frac{n+3}{2}\right\rfloor}}\left(\frac{\alpha^{n+3}-\beta^{n+3}}{\alpha-\beta}\right) e_{3} \\
= & \frac{1}{(c d)^{\left\lfloor\frac{n}{2}\right\rfloor}}\left(\frac{\alpha^{*} \alpha^{n}-\beta^{*} \beta^{n}}{\alpha-\beta}\right),
\end{aligned}
$$


and for $n$ is odd we get,

$$
\begin{aligned}
B_{n}= & \sum_{l=0}^{3} b_{n+l} e_{l} \\
= & \sum_{l=0}^{3}\left(\frac{c^{1-\xi(n+l)}}{(c d)^{\left.\frac{n+1}{2}\right\rfloor}}\right)\left(\frac{\alpha^{n+l}-\beta^{n+l}}{\alpha-\beta}\right) e_{l} \\
= & \frac{1}{(c d)^{\left\lfloor\frac{n}{2}\right\rfloor}}\left(\frac{\alpha^{n}-\beta^{n}}{\alpha-\beta}\right) e_{0}+\frac{c}{(c d)^{\left.\frac{n+1}{2}\right\rfloor}}\left(\frac{\alpha^{n+1}-\beta^{n+1}}{\alpha-\beta}\right) e_{1} \\
& +\frac{1}{(c d)^{\left.\frac{n+2}{2}\right\rfloor}}\left(\frac{\alpha^{n+2}-\beta^{n+2}}{\alpha-\beta}\right) e_{2}+\frac{c}{(c d)^{\left.\frac{n+3}{2}\right\rfloor}}\left(\frac{\alpha^{n+3}-\beta^{n+3}}{\alpha-\beta}\right) e_{3} \\
= & \frac{1}{(c d)^{\left\lfloor\frac{n}{2}\right\rfloor}}\left(\frac{\alpha^{* *} \alpha^{n}-\beta^{* *} \beta^{n}}{\alpha-\beta}\right) .
\end{aligned}
$$

Theorem 2.4. For any two nonnegative integer $n$ and $r$, with $r \leq n$, we have

$$
B_{2(n+r)} B_{2(n-r)}-B_{2 n}^{2}=\frac{\alpha^{*} \beta^{*}\left((c d)^{2 r}-\alpha^{4 r}\right)+\beta^{*} \alpha^{*}\left((c d)^{2 r}-\beta^{4 r}\right)}{(\alpha-\beta)^{2}(c d)^{2 r}} .
$$

Proof. Using the Binet formula (2.4), we obtain

$$
\begin{aligned}
B_{2(n+r)} B_{2(n-r)}-B_{2 n}^{2}= & {\left[\frac{1}{(c d)^{n+r}}\left(\frac{\alpha^{*} \alpha^{2 n+2 r}-\beta^{*} \beta^{2 n+2 r}}{\alpha-\beta}\right)\right]\left[\frac{1}{(c d)^{n-r}}\left(\frac{\alpha^{*} \alpha^{2 n-2 r}-\beta^{*} \beta^{2 n-2 r}}{\alpha-\beta}\right)\right] } \\
& -\left[\frac{1}{(c d)^{2 n}}\left(\frac{\alpha^{*} \alpha^{2 n}-\beta^{*} \beta^{2 n}}{\alpha-\beta}\right)\left(\frac{\alpha^{*} \alpha^{2 n}-\beta^{*} \beta^{2 n}}{\alpha-\beta}\right)\right] \\
= & \frac{1}{(c d)^{2 n}} \frac{1}{(\alpha-\beta)^{2}}\left[\left(\alpha^{*} \alpha^{2 n+2 r}-\beta^{*} \beta^{2 n+2 r}\right)\left(\alpha^{*} \alpha^{2 n-2 r}-\beta^{*} \beta^{2 n-2 r}\right)\right. \\
& \left.-\left(\alpha^{*} \alpha^{2 n}-\beta^{*} \beta^{2 n}\right)\left(\alpha^{*} \alpha^{2 n}-\beta^{*} \beta^{2 n}\right)\right] \\
= & \frac{1}{(\alpha \beta)^{2 n}} \frac{1}{(\alpha-\beta)^{2}}\left[\left(\alpha^{*} \alpha^{2 n}\right)^{2}-\alpha^{*} \beta^{*} \alpha^{2 n+2 r} \beta^{2 n-2 r}-\alpha^{*} \beta^{*} \alpha^{2 n-2 r} \beta^{2 n+2 r}\right. \\
& \left.+\left(\beta^{*} \beta^{2 n}\right)^{2}-\left(\alpha^{*} \alpha^{2 n}\right)^{2}+2 \alpha^{*} \alpha^{2 n} \beta^{*} \beta^{2 n}-\left(\beta^{*} \beta^{2 n}\right)^{2}\right] \\
= & \frac{1}{(\alpha-\beta)^{2}}\left[\alpha^{*} \beta^{*}\left(1-\left(\frac{\alpha}{\beta}\right)^{2 r}\right)+\beta^{*} \alpha^{*}\left(1-\left(\frac{\beta}{\alpha}\right)^{2 r}\right)\right] \\
= & \frac{\alpha^{*} \beta^{*}\left((c d)^{2 r}-\alpha^{4 r}\right)+\beta^{*} \alpha^{*}\left((c d)^{2 r}-\beta^{4 r}\right)}{(\alpha-\beta)^{2}(c d)^{2 r}} .
\end{aligned}
$$

Theorem 2.5. For any nonnegative integer $n$, we have

$$
B_{2(n+1)} B_{2(n-1)}-B_{2 n}^{2}=\frac{\alpha^{*} \beta^{*}\left((c d)^{2}-\alpha^{4}\right)+\beta^{*} \alpha^{*}\left((c d)^{2}-\beta^{4}\right)}{(\alpha-\beta)^{2}(c d)^{2}} .
$$

Proof. In the previous theorem, if we take $r=1$ we get the proof. 
Theorem 2.6 (Catalan's Identity). For nonnegative integer $n$ and nonnegative even integer $r$, with $r \leq n$, we have

$$
B_{n+r} B_{n-r}-B_{n}^{2}=\left\{\begin{array}{cc}
\frac{\alpha^{*} \beta^{*}\left((c d)^{r}-\alpha^{2 r}\right)+\beta^{*} \alpha^{*}\left((c d)^{r}-\beta^{2 r}\right)}{(c d)^{r}(\alpha-\beta)^{2}}, & \text { if } n \text { is even } \\
\frac{\alpha^{* *} \beta^{* *}\left((c d)^{r}-\alpha^{2 r}\right)+\beta^{* *} \alpha^{* *}\left((c d)^{r}-\beta^{2 r}\right)}{(c d)^{r-1}(\alpha-\beta)^{2}}, & \text { if } n \text { is odd } .
\end{array}\right.
$$

Proof. By using the Binet formula (2.4), for $n$ is even we get,

$$
\begin{aligned}
B_{n+r} B_{n-r}-B_{n}^{2}= & {\left[\frac{1}{\left.(c d)^{\frac{n+r}{2}}\right\rfloor}\left(\frac{\alpha^{*} \alpha^{n+r}-\beta^{*} \beta^{n+r}}{\alpha-\beta}\right)\right]\left[\frac{1}{(c d)^{L^{\frac{n-r}{2}}}}\left(\frac{\alpha^{*} \alpha^{n-r}-\beta^{*} \beta^{n-r}}{\alpha-\beta}\right)\right] } \\
& -\left[\frac{1}{\left.(c d)^{\frac{n}{2}}\right\rfloor}\left(\frac{\alpha^{*} \alpha^{n}-\beta^{*} \beta^{n}}{\alpha-\beta}\right)\right]^{2} \\
= & \frac{1}{(c d)^{n}} \frac{1}{(\alpha-\beta)^{2}}\left[\left(\alpha^{*} \alpha^{n+r}-\beta^{*} \beta^{n+r}\right)\left(\alpha^{*} \alpha^{n-r}-\beta^{*} \beta^{n-r}\right)\right] \\
& -\frac{1}{(c d)^{n}} \frac{1}{(\alpha-\beta)^{2}}\left(\alpha^{*} \alpha^{n}-\beta^{*} \beta^{n}\right)^{2} \\
= & \frac{1}{(c d)^{n}} \frac{1}{(\alpha-\beta)^{2}}\left[\alpha^{*} \beta^{*}(\alpha \beta)^{n}\left(1-\left(\frac{\alpha}{\beta}\right)^{r}\right)+\beta^{*} \alpha^{*}(\alpha \beta)^{n}\left(1-\left(\frac{\beta}{\alpha}\right)^{r}\right)\right] \\
= & \frac{\alpha^{*} \beta^{*}\left((c d)^{r}-\alpha^{2 r}\right)+\beta^{*} \alpha^{*}\left((c d)^{r}-\beta^{2 r}\right)}{(c d)^{r}(\alpha-\beta)^{2}},
\end{aligned}
$$

and for $n$ is odd, we get

$$
\begin{aligned}
B_{n+r} B_{n-r}-B_{n}^{2}= & {\left[\frac{1}{(c d)^{L^{\left.\frac{n+r}{2}\right\rfloor}}}\left(\frac{\alpha^{* *} \alpha^{n+r}-\beta^{* *} \beta^{n+r}}{\alpha-\beta}\right)\right]\left[\frac{1}{(c d)^{\left.\frac{n-r}{2}\right\rfloor}}\left(\frac{\alpha^{* *} \alpha^{n-r}-\beta^{* *} \beta^{n-r}}{\alpha-\beta}\right)\right] } \\
& -\left[\frac{1}{(c d)^{\left\lfloor\frac{n}{2}\right\rfloor}}\left(\frac{\alpha^{* *} \alpha^{n}-\beta^{* *} \beta^{n}}{\alpha-\beta}\right)\right]^{2} \\
= & \frac{1}{(c d)^{n-1}} \frac{1}{(\alpha-\beta)^{2}}\left[\left(\alpha^{* *} \alpha^{n+r}-\beta^{* *} \beta^{n+r}\right)\left(\alpha^{* *} \alpha^{n-r}-\beta^{* *} \beta^{n-r}\right)\right] \\
& -\frac{1}{(c d)^{n-1}} \frac{1}{(\alpha-\beta)^{2}}\left(\alpha^{* *} \alpha^{n}-\beta^{* *} \beta^{n}\right)^{2} \\
= & \frac{1}{(c d)^{n-1}} \frac{1}{(\alpha-\beta)^{2}}\left[\alpha^{* *} \beta^{* *}(\alpha \beta)^{n}\left(1-\left(\frac{\alpha}{\beta}\right)^{r}\right)+\beta^{* *} \alpha^{* *}(\alpha \beta)^{n}\left(1-\left(\frac{\beta}{\alpha}\right)^{r}\right)\right] \\
= & \frac{\alpha^{* *} \beta^{* *}\left((c d)^{r}-\alpha^{2 r}\right)+\beta^{* *} \alpha^{* *}\left((c d)^{r}-\beta^{2 r}\right)}{(c d)^{r-1}(\alpha-\beta)^{2}} .
\end{aligned}
$$

Theorem 2.7. For any integer $n \geq 0$, the following properties hold;

$$
\begin{gathered}
B_{n}+\bar{B}_{n}=2 b_{n}, \\
B_{n}^{2}+B_{n} \bar{B}_{n}=2 b_{n} B_{n}, \\
B_{n+2}=6 B_{n+1}-B_{n} .
\end{gathered}
$$


Proof. By using bi-periodic balancing number, quaternions and the definition of conjugate of quaternions we get,

$$
\begin{aligned}
& B_{n}+\overline{B_{n}}=\sum_{l=0}^{3} b_{n+l} e_{l}+\left(\overline{\sum_{l=0}^{3} b_{n+l} e_{l}}\right) \\
&=b_{n} e_{0}+b_{n+1} e_{1}+b_{n+2} e_{2}+b_{n+3} e_{3}+b_{n} \overline{e_{0}}+b_{n+1} \overline{e_{1}}+b_{n+2} \overline{e_{2}}+b_{n+3} \overline{e_{3}} \\
&=2 b_{n}+e_{1}\left(b_{n+1}-b_{n+1}\right)+e_{2}\left(b_{n+2}-b_{n+2}\right)+e_{3}\left(b_{n+3}-b_{n+3}\right) \\
&=2 b_{n}, \\
& B_{n}^{2}+B_{n} \bar{B}_{n}=\left(\sum_{l=0}^{3} b_{n+1} e_{l}\right)^{2}+\sum_{l=0}^{3} b_{n+l} e_{l}\left(\overline{\sum_{l=0}^{3} b_{n+l} e_{l}}\right) \\
&= b_{n}^{2} e_{0}^{2}+b_{n} b_{n+1} e_{0} e_{1}+b_{n} b_{n+2} e_{0} e_{2}+b_{n} b_{n+3} e_{0} e_{3} \\
&+b_{n+1} b_{n} e_{1} e_{0}+b_{n+1}^{2} e_{1}^{2}+b_{n+1} b_{n+2} e_{1} e_{2}+b_{n+1} b_{n+3} e_{1} e_{3} \\
&+b_{n+2} b_{n} e_{2} e_{0}+b_{n+2} b_{n+1} e_{2} e_{1}+b_{n+2}^{2} e_{2}^{2}+b_{n+2} b_{n+3} e_{2} e_{3} \\
&+b_{n+3} b_{n} e_{3} e_{0}+b_{n+3} b_{n+1} e_{3} e_{1}+b_{n+3} b_{n+2} e_{3} e_{2}+b_{n+3}^{2} e_{3}^{2} \\
&+b_{n}^{2} e_{0} \overline{e_{0}}+b_{n} b_{n+1} e_{0} \overline{e_{1}}+b_{n} b_{n+2} e_{0} \overline{e_{2}}+b_{n} b_{n+3} e_{0} \overline{e_{3}} \\
&+b_{n+1} b_{n} e_{1} \overline{e_{0}}+b_{n+1}^{2} e_{1} \overline{e_{1}}+b_{n+1} b_{n+2} e_{1} \overline{e_{2}}+b_{n+1} b_{n+3} e_{1} \overline{e_{3}} \\
&+b_{n+2} b_{n} e_{2} \overline{e_{0}}+b_{n+2} b_{n+1} e_{2} \overline{e_{1}}+b_{n+2}^{2} e_{2} \overline{e_{2}}+b_{n+2} b_{n+3} e_{2} \overline{e_{3}} \\
&+b_{n+3} b_{n} e_{3} \overline{e_{0}}+b_{n+3} b_{n+1} e_{3} \overline{e_{1}}+b_{n+3} b_{n+2} e_{3} \overline{e_{2}}+b_{n+3}^{2} e_{3} \overline{e_{3}} \\
&= 2 b_{n} b_{n} e_{0}+2 b_{n} b_{n+1} e_{1}+2 b_{n} b_{n+2} e_{2}+2 b_{n} b_{n+3} e_{3} \\
&= 2 b_{n} B_{n}
\end{aligned}
$$

and

$$
\begin{aligned}
6 B_{n+1}-B_{n} & =6 \sum_{l=0}^{3} b_{(n+1)+l} e_{l}-\sum_{l=0}^{3} b_{n+l} e_{l} \\
& =6 b_{n+1} e_{o}+6 b_{n+2} e_{1}+6 b_{n+3} e_{2}+6 b_{n+4} e_{3}-b_{n} e_{0}-b_{n+1} e_{1}-b_{n+2} e_{2}-b_{n+3} e_{3} \\
& =\left(6 b_{n+1} e_{o}-b_{n} e_{0}\right)+\left(6 b_{n+2} e_{1}-b_{n+1} e_{1}\right)+\left(6 b_{n+3} e_{2}-b_{n+2} e_{2}\right)+\left(6 b_{n+4} e_{3}-b_{n+3} e_{3}\right) \\
& =b_{n+2} e_{0}+b_{n+3} e_{1}+b_{n+4} e_{2}+b_{n+5} e_{3} \\
& =\sum_{l=0}^{3} b_{(n+2)+l} e_{l} \\
& =B_{n+2} .
\end{aligned}
$$

\section{CONFLICTS OF INTEREST}

The authors declare that there are no conflicts of interest regarding the publication of this article. 


\section{REFERENCES}

[1] Behera, A., Panda, G.K., On the square roots of triangular numbers, Fibonacci Quarterly, 37(2)(1999), 98-105. 1

[2] Edson, M., Yayenie, O., A new generalization of Fibonacci sequences and extended Binet's formula, Integers, 9(6)(2009), 639-654. 1

[3] Halıc1, S., On Fibonacci quaternions, Advances in Applied Clifford Algebras, 22(2)(2012), 321-327. 1

[4] Hamilton, W.R., Lectures on quaternions, Dublin, 1853. 1

[5] Horadam, A.F., Complex Fibonacci numbers and Fibonacci quaternions, The American Mathematical Monthly, 70(3)(1963), 289-291. 1

[6] Iyer, M.R., Some results on Fibonacci quaternions, Fibonacci Quarterly, 7(2)(1969), 201-210. 1

[7] Jordan, J.H., Gaussian Fibonacci and Lucas numbers, Fibonacci Quarterly, 3(1965), 315-318. 1

[8] Koshy, T., Fibonacci and Lucas numbers with applications, Wiley, New York, 2001. 1

[9] Ozkan Kiz1lırmak, G., Tasc1, D., Expression of reciprocal sum of Gaussian Lucas sequences by Lambert series, Journal of Science and Arts, 48(3)(2019), 587-592. 1

[10] Tan, E., Yılmaz, S., Sahin, M., A note on bi-periodic Fibonacci and Lucas quaternions, Chaos, Solitions and Fractals, 85(2016), 138-142. 1

[11] Tasc1, D., Ozkan Kızılırmak, G., On the periods of bi-periodic Fibonacci and bi-periodic Lucas numbers, Discrete Dynamics in Nature and Society, (2016), 1-5. 1 